\title{
Assessment of the HPA Axis: Another New Test?
}

\author{
Ashley Grossman ${ }^{1}$
}

Received: 28 July 2015/Accepted: 22 August 2015/Published online: 11 September 2015

(C) Springer Science+Business Media New York 2015

As the pituitary fails, each axis appears to show defects and then ultimately fails in a generally predictable order, with a sense that the least important hormones decrease first with the most critical hormonal axes only being lost when pituitary damage is at a maximum. There are exceptions, as in lymphocytic hypophysitis, but this is the exception which proves the rule. Thus, it is uncommon for pituitary disease to cause failure of the hypothalamo-pituitaryadrenal (HPA) axis, but when it does fail, it can be catastrophic. Pituitary ACTH, under the joint control of hypothalamic $\mathrm{CRH}$ and vasopressin, causes the release of adrenal corticosteroids, specifically and most critically corticosterone in rodents and cortisol in the human, while mineralocorticoids such as aldosterone are principally regulated by the renin-angiotensin system. Cortisol in humans is secreted in a circadian rhythm with maximum circulating levels at 7-9 a.m. and a nadir around midnight. However, most importantly, cortisol is a stress hormone which is stimulated by severe cardiovascular, infectious, and-to some extent-psychological stress. An absent or poor glucocorticoid response to stress causes cardiovascular collapse and may be lethal. Thus, any form of severe pituitary damage, most usually pituitary tumors but including hypothalamic disease, radiotherapy, and surgical intervention, may cause a failure of HPA responsiveness to severe stress (such as trauma, severe infection, or surgery) leading to cardiovascular collapse and death: hence, assessment of the HPA axis is crucial in monitoring

Ashley Grossman

ashley.grossman@ocdem.ox.ac.uk

1 Oxford Centre for Diabetes, Endocrinology and Metabolism, Churchill Hospital, University of Oxford, Oxford OX3 7LE, UK patients with disorders of the pituitary and hypothalamus, and judging adequate replacement regimes [1].

In terms of attempts to assess the HPA axis in patients with such tumors, there has been some confusion as to what exactly is the criterion for 'normality.' The gold standard has been the insulin tolerance test (ITT), as this examines the entire HPA axis. The original studies compared the ITT to the cortisol responses to surgery, suggesting that investigating the lower limit of the normal stress response in severe surgical stress, and comparing that to the ITT, would enable one to establish a minimum cortisol which was both 'normal' and 'safe' [2]. The ITT involves careful oversight and experience in its use; it is contraindicated where there is a history of cerebral or cardiac dysrhythmias and should only be performed in units where the necessary expertise is available. Some would also avoid its use in the elderly. Thus, other dynamic tests have been studied. However, subsequent studies have simply compared patients in terms of whether their peak cortisol response was within the normal range for that test, and for the most part have not considered whether this would necessarily render patients safe in terms of being able to respond to a severe stressor without the need for additional corticosteroids. Some use the glucagon stimulation test, especially in those with contraindications to the ITT, or in children. Different cut-offs need to be used for the glucagon test compared to the ITT, and its mechanism of action is even less clear than for the ITT, although it shares with the ITT the ability to also assess growth hormone reserve [3]. In my opinion, it is a longer and less well-tolerated test as compared to the ITT, and less liked by patients. Another alternative is the corticotrophin releasing hormone (CRH) test, using intravenous human or ovine-sequence $\mathrm{CRH}$ : this directly tests pituitary ACTH function rather then the whole HPA axis, but I tend to agree with a recent opinion 
that 'the CRH test has not generally been regarded as a useful tool in the clinical setting' [4]. The short corticotropin stimulation test, usually referred to as Synacthen or Cosyntropin in many areas, has been extensively utilized as a surrogate marker of adrenocortical reserve, with normal responses established from a normative population rather then from the ability of a patient to respond to, e.g., major surgery. Nevertheless, the close correlation of the SST with the ITT has established this test to be the standard assessment in many neuroendocrine clinics, including that of the author, as long as there is sufficient time between the insult to the HPA axis and the SST for them to be correlated [5]; thus, performing the SST soon after pituitary surgery is valueless and dangerous [6]. The low-dose ST, using $1 \mu \mathrm{g}$, may be probably slightly more sensitive as a marker but is cumbersome to use, and it is technically difficult to achieve reproducible dilutions and may need to have 60 as well as $30 \mathrm{~min}$ samples [7]: it has not achieved widespread acceptance. One further caveat is that the cortisol assay used is critical in determining the normal range [8], a fact that is often not appreciated in many textbooks.

Nevertheless, there remains the need for a test which fully assesses the complete HPA axis, and which may be used where there is not the necessary supervision for the ITT. Many dynamic tests have been suggested, but as noted above, to date only the glucagon test has been in widespread use. The CRH test acts directly on the pituitary and does not assess the whole HPA axis. Thus, Gasco et al. have explored the use of acylated ghrelin as a provocative test of the HPA axis: is it safe and accurate [9]? Ghrelin secretagogues not only directly stimulate the pituitary corticotrophs but also act via hypothalamic pathways, such that stalk section abolishes the cortisol response to such agents. So, the question the authors ask is whether the use of this agent, which is well tolerated with few side effects, can add information over and above basal cortisol levels. They investigated 49 patients with pituitary disease and compared the responses of ACTH and cortisol to acylated ghrelin, low-dose ACTH, metyrapone, and the ITT: quite a feat and admirably comprehensive. In addition, rather than using pre-established cut-offs, they established ROCs for each test procedure. My one criticism of this otherwise very complete study is that the 'gold standard' used as the criterion of an abnormality was not uniform and varied between the ITT in 28 patients to the use of metyrapone (19 patients) and low-does SST (2 patients). And the outcome? Acylated ghrelin was certainly well tolerated, with only some facial flushing seen in some patients. In the event, while the acylated ghrelin was concluded to show 'good diagnostic accuracy,' it was no better than a basal $09.00 \mathrm{~h}$ serum cortisol.
These authors are to be congratulated in carrying out an important study in a clinically significant area, and also for being honest in their appraisal that their new test does not offer any significant advantages. So, where does that leave the clinician with the daily problem in assessing patients with pituitary disease for adrenocortical deficiency? It is likely that other tests, such as the $\mathrm{CRH}$ and glucagon tests, will remain in widespread use, but I believe that the ITT will retain its place after some 50 years as the diagnostic test of choice, with the SST as the everyday workhorse for patients without any recent insult. But a basal cortisol level, cheap and convenient, remains the simple test of primary choice, as has been known for many years.

\section{Compliance with Ethical Standards}

Conflict of interest The author confirms he has no conflict of interest.

\section{References}

1. A.B. Grossman, The diagnosis and management of central hypoadrenalism. J. Clin. Endocrinol. Metab. 95, 4855-4863 (2010)

2. F.S. Plumpton, G.M. Besser, The adrenocortical response to surgery and insulin-induced hypoglycaemia in corticosteroidtreated and normal subjects. Br. J. Surg. 56, 216-219 (1969)

3. Y. Simsek, Z. Karaca, F. Tanriverdi, K. Unluhizarci, A. Selcukli, F. Kelestimur, A comparison of low-dose ACTH, glucagon stimulation and insulin tolerance test in patients with pituitary disorders. Clin. Endocrinol. (Oxf). 82, 45-52 (2015)

4. M. Maghnie, E. Uga, F. Temporini, N. Di Iorgi, A. Secco, C. Tinelli, A. Papalia, M.R. Casini, S. Loche, Evaluation of adrenal function in patients with growth hormone deficiency and hypothalamic-pituitary disorders; comparison between insulin-induced hypoglycemia, low-dose ACTH standard ACTH and CRH stimulation tests. Eur. J. Endocrinol. 152, 735-741 (2005)

5. A. Agha, J.W. Tomlinson, P.M. Clark, G. Holder, P.M. Stewart, The long-term predictive accuracy of the short synacthen (corticotropin) stimulation test for assessment of the hypothalamicpituitary-adrenal axis. J. Clin. Endocrinol. Metab. 91, 43-47 (2006)

6. J.J. Mukherjee, J.J. de Castro, G. Kaltsas, F. Afshar, A.B. Grossman, J.A. Wass, G.M. Besser, A comparison of the insulin tolerance/glucagon test with the short ACTH stimulation test in the assessment of the hypothalamo-pituitary-adrenal axis in the early post-operative period after hypophysectomy. Clin. Endocrinol. (Oxf). 47, 51-60 (1997)

7. J. Cartaya, M. Misra, The low-dose ACTH stimulation tests: is 30 minutes long enough? Endocr. Pract. 21, 508-513 (2015)

8. N. El-Farhan, A. Picket, D. Ducro, C. Bailey, K. Mitchem, N. Morgan, A. Armston, L. Jones, C. Evans, D.A. Rees, Methodspecific serum cortisol responses to the adrenocorticotrophin test: comparison of gas chromatography-mass spectrometry and five automated immunoassays. Clin. Endocrinol. (Oxf). 78, 673-680 (2013)

9. V. Gasco, A. Berton, M.P. Caprino, I. Karamouzis, M. Maccario, E. Ghigo, S. Grottoli, Acylated ghrelin as provocative test for the diagnosis of ACTH deficiency in patients with hypothalamuspituitary disease. Endocrine (2014). doi:10.1007/s12020-0140494-5 